\title{
DQ thermal buckling analysis of embedded curved carbon nanotubes based on nonlocal elasticity theory
}

Abstract

To investigate the thermal buckling of curved carbon nanotubes (CCNTs) embedded in an elastic medium, nonlocal elasticity theory is employed in combination with the theory of thin curved beams. Differential quadrature (DQ) method is implemented to discretize the resulted governing equations. Solving these equations enables us to estimate the critical temperature and the critical axial buckling load for CCNTs surrounded by an elastic medium and under the effect of a uniform temperature change. The elastic interaction between the nanotube and its surrounding medium is modeled as a Winkler-Pasternak elastic foundation. The fast convergence of the DQ method is demonstrated and also its accuracy is verified by comparing the results with available solutions in the literature. The effects of various parameters such as different boundary conditions, nonlocal parameter, Winkler and Pasternak elastic modulus, temperature and nanotube curvature on the critical buckling temperature and load are successfully studied. The results reveal that the critical buckling load depends significantly on the curvature of the CCNT.

\section{Keywords}

Curved carbon nanotubes; thermal buckling; nonlocal elasticity theory; differential quadrature method.

\section{AliReza Setoodeh ${ }^{\mathrm{a}^{*}}$ \\ Morteza Derahaki ${ }^{\text {b }}$ \\ Navid Bavi ${ }^{\mathrm{c}}$}

${ }^{a}$ Associate Professor, Faculty of Mechanical \& Aerospace Engineering, Shiraz University of Technology, Shiraz, Iran

${ }^{\mathrm{b}}$ Faculty of Mechanical \& Aerospace

Engineering, Shiraz University of Technology,

Shiraz, Iran

${ }^{c^{\dagger}}$ Mechanical Engineering Department, Faculty

of Engineering, Shahid Chamran University,

Ahvaz, Iran

${ }^{\dagger}$ Current address: Mechanosensory Biophysics

Laboratory, Victor Chang Cardiac Research

Institute, Sydney, New SouthWales 2010,

Australia

Corresponding author:

$\mathrm{a}^{*}$ setoodeh@sutech.ac.ir

http://dx.doi.org/10.1590/1679-78251894

Received 01.02.2015

Accepted 19.03.2015

Available online 02.05.2015

\section{INTRODUCTION}

Nano-electro-mechanical systems (NEMS) are among fast growing technologies which deal with the production of nano-scale machines. These devices are being extensively used in many advanced industries such as aerospace, automotive, biotechnology, and audiometric equipment (Dai et al., 1996). Among the nanostructures, carbon nanotubes (CNTs) are one of the most important structures 
which are regarded as representatives for nanotechnology due to their unique characteristics. These structures have outstanding mechanical, physical and chemical properties such as high strength, high stiffness to weight ratio, as well as high electrical and thermal conductivity (Ruoff and Lorents, 1995; Tans et al., 1998; Tombler et al., 2000; Jiang et al., 2004; Friedman et al., 2005; Sedighi et al., 2014).

Most of the potential applications of CNTs dramatically depend on their mechanical performance including their buckling behavior due to the thermal effects and the environment they are embedded in. As in reality, there are no perfect straight CNTs, thus it is imperative to assess buckling behavior of curved CNTs. More importantly, CCNTs can be used as single-electron transistor (Postma et al., 2001), mechanical nanosprings and nanocoils (Liu et al., 2014). So far, several experimental methods (Iijima et al., 1996; Falvo et al., 1997; Waters et al., 2005) and molecular dynamics simulation approaches (Yakobson et al., 1996; Ozaki et al., 2000; Liew et al., 2004; Liu et al., 2004; Wang et al., 2005; Cao and Chen, 2006; Kulathunga et al., 2010; Li et al., 2013) have been carried out to accurately examine the corresponding mechanical response of nanostructures. However, despite of the considerable advances in nanotechnology, experimental tests are difficult to be performed and handled at nano-scale and also, all-atom molecular dynamics simulation method is computationally expensive. Hence, introducing theoretical models (e.g. modified continuum mechanics approaches) is required as they are relatively simple to be applied with less time-consuming without compromising the accuracy of the results.

Eringen (1983) proposed the nonlocal elasticity theory which has been widely used for continuum mechanics modeling of CNTs (Wang et al., 2008; Murmu and Pradhan, 2010; Wang et al., 2010; Murmu and Adhikari, 2011; Narendar and Gopalakrishnan, 2012; Thai, 2012; Thai and Vo, 2012; Şimşek and Yurtcu, 2013; Tounsi et al., 2013, Asemi et al., 2014). In this theory, the effects of small scaling parameter on the mechanical analysis of nanostructures are taken into account. The main idea of the nonlocal elasticity theory is that the stress at an arbitrary point of a body is a function of the strain at all over the body (Eringen, 1983). Subsequently, Reddy (2007) reformulated different beam theories based on the nonlocal beam theory. He employed the nonlocal theory to investigate the bending, buckling, and vibration of nanobeams. Civalek and Demir (2011) used an Euler-Bernoulli beam model based nonlocal elasticity theory to examine the static and buckling analysis of cantilever CNTs. They numerically solved the governing equations and showed that the nonlocal parameter has a significant effect on the static and buckling behavior of CNTs. Wang et al. (2008) examined the effects of thermal loading on the vibration and buckling of CNTs conveying fluid. Rastgo et al. (2005) investigated instability of curved beams made of functionally graded material under thermal loading. Murmu and Pradhan (2010) studied the effects of thermal loading on the buckling load of CNTs embedded in an elastic medium. They modeled the elastic medium around the nanotubes as a singlevariable Winkler elastic medium. Wang et al. (2010) assessed the effects of small scaling parameter on the thermal buckling of CNTs. Murmu and Adhikari (2011) analytically investigated the stability of a system consisting of two nanobeams.

In this paper, the thermal buckling analysis of curved CNTs resting on an elastic medium is investigated. Despite the significance, there are not many researches previously conducted on curved CNTs, whereas to the best of the authors' knowledge, the present study is the first to investigate the thermal buckling of curved CNTs embedded in an elastic medium employing DQ method. At first, the critical buckling temperature of CNTs resting on the elastic medium is calculated. Afterwards, 
the critical buckling load of CCNTs embedded in an elastic medium and under the effect of a uniform temperature alteration is estimated. In these analyses, the elastic medium around the nanotube is modeled as a two-variable Winkler-Pasternak elastic medium. The governing equations are developed based on the thin curved beams and nonlocal elasticity theories. Using powerful numerical method of differential quadrature method, the critical buckling load is calculated for different boundary conditions. The effects of various parameters such as boundary conditions, small scaling parameter, Winkler and Pasternak parameters, temperature change and angle of curvature of nanotubes on the critical buckling load are examined.

\section{NONLOCAL ELASTICITY THEORY OF CCNTS}

To derive the governing equations of the thermal buckling of a CCNT embedded in an elastic medium, a combination of the thin curved beam and nonlocal elasticity theories are employed. A curved beam is illustrated in Figure 1, where $L$ is the length of the beam. The orientation of the mid-plane is determined by the polar component of $\alpha$.

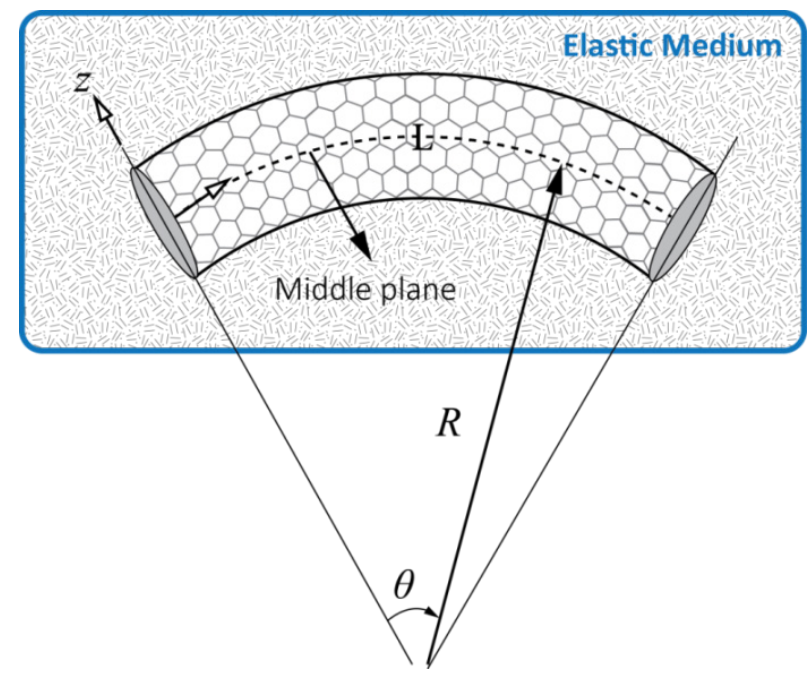

Figure 1: Schematic of a portion of a CCNT.

The relation between this component and the angle of curvature of beam, $\theta$ is as follows;

$$
\alpha=R \theta
$$

where $R$ is the radius of curvature of the beam.

The kinematics relations of thin curved beams are derived based on the kinematics of thin-walled shells. This can be done by neglecting the out of plane components as well as their derivatives. Hence, the strain of each point on the beam is obtained based on the kinematics equations of thin curved beams which is stated as follows (Qatu, 2004).

$$
\varepsilon=\varepsilon_{0}+z \kappa
$$




$$
\begin{gathered}
\varepsilon_{0}=\frac{\partial u_{0}}{\partial \alpha}+\frac{w_{0}}{R} \\
\kappa=-\frac{\partial^{2} w_{0}}{\partial \alpha^{2}}+\frac{1}{R} \frac{\partial u_{0}}{\partial \alpha}
\end{gathered}
$$

where $u_{0}$ and $w_{0}$, are the displacement components of a material point on the mid-plane in the polar directions of $\alpha$ and $z$, respectively. $\varepsilon_{0}$ is the strain arising from the mid-plane stretching, and $\kappa$ is the curvature of the beam due to bending.

The modified elasticity theories have enabled us to fill the present gap between molecular dynamics and continuum mechanics. Eringen's nonlocal theory states that the stress at an arbitrary physical point of a body is assumed to be a function of the strain field for the whole body. The differential form of this theory is as follows (Eringen, 1983);

$$
\left(1-\mu \nabla^{2}\right) \sigma_{i j}=C_{i j m n} \varepsilon_{m n}
$$

where $C$ and $\nabla$ represent the stiffness matrix of the structure and the gradient operator, respectively. Also, $\mu$ is the nonlocal parameter.

$$
\mu=\left(e_{0} a\right)^{2}
$$

$e_{0}$ is a constant value assigned to the body and $a$ represents the internal characteristic length. In general, $e_{0} a$ is believed to be less than 2 for the case of CNTs (Setoodeh et al., 2011; Malekzadeh et al., 2012). A homogeneous isotropic nanotube is assumed here while an axial load is perpendicularly exerted on the nanotube cross section. Thus, Eq. (6) is simplified for an isotropic and homogeneous nanotube based on the thin beam theory, where the effect of shear deformation is neglected. Hence, the stress-strain relation for the aforementioned type of loading is modified as,

$$
\left(1-\mu \nabla^{2}\right) \sigma=E \varepsilon
$$

where $\sigma$ and $\varepsilon$ represent the normal nonlocal stress and strain at an arbitrary point, respectively, and $E$ denotes the Young's modulus of the nanotube. The stress resultants are the integrals of stress over the cross section area of the nanotube. Therefore, the nonlocal stress resultant are given by

$$
\begin{aligned}
& \hat{N}=\int_{A} \sigma \mathrm{d} A \\
& \hat{M}=\int_{A} \sigma z \mathrm{~d} A
\end{aligned}
$$

where $A$ represents the cross section area of the nanotube. Integrating Eq. (7) over the nanotube cross section and substituting Eqs. (3) and (8) into Eq. (7), yields to 


$$
\hat{N}-\mu \frac{\partial^{2} \hat{N}}{\partial \alpha^{2}}=E A \varepsilon_{0}
$$

Multiplying Eq. (7) by $z$ and integrating the result over the cross section area, and substituting Eqs. (4) and (9) leads to find another constitutive equation in terms of stress resultants as bellow

$$
\hat{M}-\mu \frac{\partial^{2} \hat{M}}{\partial \alpha^{2}}=E I \kappa
$$

where $I$ represents the moment of inertia of the nanotube cross section.

\section{GOVERNING EQUATIONS OF CCNTS}

In this part of the paper, the governing equations are developed using Hamilton's principle. Since the equilibrium equations for the local and nonlocal elasticity theories are the same, the local elasticity form of these equations is employed and the effect of nonlocal parameter is ignored for the sake of simplicity. The medium surrounding the nanotube is modeled as an elastic foundation with $k_{w}$ and $k_{p}$ as Winkler and Pasternak constants, respectively. It is considered that the CCNT is under the effect of a uniform temperature change of $\Delta T$ relative to the reference temperature. The resulted thermal stress due to this temperature variation is considered as an external load. Based on the theory of thermo-elasticity, the value of this force is calculated as

$$
F_{t h}=\frac{E A}{1-2 \nu} \alpha_{t h} \Delta T
$$

$\alpha_{t h}$ and $\nu$ represent the thermal expansion coefficient and the Poisson's ratio of the nanotube, respectively. For the sake of simplicity, the following variable is introduced.

$$
\alpha^{\prime}=\frac{\alpha_{t h}}{1-2 \nu}
$$

Thus, Eq. (12) yields to

$$
F_{t h}=E A \alpha^{\prime} \Delta T
$$

In order to present a comprehensive study, it is also assumed that the CNT is under an axial load, $P$. Therefore, the strain energy of the nanotube, the potential energy of the elastic medium and the work done due to the thermal loading and external force can be represented by Eqs. (15)-(18), respectively.

$$
U_{s}=\frac{1}{2} \int_{V} \sigma \varepsilon \mathrm{d} V
$$




$$
\begin{gathered}
U_{f}=\frac{1}{2} \int_{0}^{\theta}\left[k_{w} w_{0}^{2}+k_{p}\left(\frac{\partial w_{0}}{\partial \alpha}\right)^{2}\right] \mathrm{d} \alpha \\
W_{e x t}^{t h}=\frac{1}{2} \int_{0}^{\theta} F_{t h}\left(\frac{\partial w_{0}}{\partial \alpha}\right)^{2} \mathrm{~d} \alpha \\
W_{e x t}^{P}=\frac{1}{2} \int_{0}^{\theta} P\left(\frac{\partial w_{0}}{\partial \alpha}\right)^{2} \mathrm{~d} \alpha
\end{gathered}
$$

where $V$ is the total volume of the nanotube. Using Hamilton's principle,

$$
\delta \int_{t}\left(U_{s}+U_{f}-W_{e x t}^{t h}-W_{e x t}^{P}\right) \mathrm{d} t=0
$$

And, applying variation principle to the functional forms stated in Eqs. (15)-(18) and performing some manipulations, the equilibrium equations and the boundary conditions are obtained as follows

$$
\begin{gathered}
\frac{\partial N}{\partial \alpha}+\frac{1}{R} \frac{\partial M}{\partial \alpha}=0 \\
\frac{N}{R}-\frac{\partial^{2} M}{\partial \alpha^{2}}+k_{w} w_{0}-k_{p} \frac{\partial^{2} w_{0}}{\partial \alpha^{2}}+F_{t h} \frac{\partial^{2} w_{0}}{\partial \alpha^{2}}+P \frac{\partial^{2} w_{0}}{\partial \alpha^{2}}=0
\end{gathered}
$$

Eqs. (20) and (21) are the equilibrium equations, and Eqs. (22)-(24) represent different boundary conditions.

$$
\begin{gathered}
u_{0}=0 \quad \text { or } \quad N+\frac{M}{R}=0 \\
M=0 \quad \text { or } \quad \frac{\partial w_{0}}{\partial \alpha}=0 \\
w_{0}=0 \quad \text { or } \quad \frac{\partial M}{\partial \alpha}+k_{p}\left(\frac{\partial w_{0}}{\partial \alpha}\right)-F_{t h}\left(\frac{\partial w_{0}}{\partial \alpha}\right)-P\left(\frac{\partial w_{0}}{\partial \alpha}\right)=0
\end{gathered}
$$

The boundary conditions for the simply supported condition are obtained as,

$$
\begin{aligned}
& w_{0}=0 \\
& N+\frac{M}{R}=0 \\
& M=0
\end{aligned}
$$

and for a clamped end it is represented as, 


$$
\begin{aligned}
& w_{0}=0 \\
& u_{0}=0 \\
& \frac{\partial w_{0}}{\partial \alpha}=0
\end{aligned}
$$

By combining the equilibrium equations (20)-(21) with Eqs. (10)-(11), the governing equations are derived as,

$$
\begin{gathered}
\left(E A+\frac{E I}{R^{2}}\right) \frac{\partial^{2} u_{0}}{\partial \alpha^{2}}+\left(\frac{E A}{R}\right) \frac{\partial w_{0}}{\partial \alpha}-\left(\frac{E I}{R}\right) \frac{\partial^{3} w_{0}}{\partial \alpha^{3}}=0 \\
\left(\frac{E A}{R}\right) \frac{\partial u_{0}}{\partial \alpha}-\left(\frac{E I}{R}\right) \frac{\partial^{3} u_{0}}{\partial \alpha^{3}}+\left(\frac{E A}{R^{2}}+k_{w}\right) w_{0}+\left(F_{t h}-k_{p}-\mu k_{w}\right) \frac{\partial^{2} w_{0}}{\partial \alpha^{2}}+ \\
+\left(E I+\mu k_{p}-\mu F_{t h}\right) \frac{\partial^{4} w_{0}}{\partial \alpha^{4}}-\mu P \frac{\partial^{4} w_{0}}{\partial \alpha^{4}}+P \frac{\partial^{2} w_{0}}{\partial \alpha^{2}}=0
\end{gathered}
$$

To simplify the equations, the following nondimensional parameters are introduced,

$$
\beta=\frac{\alpha}{L}, \quad \theta=\frac{L}{R}, \quad K_{w}=\frac{k_{w} L^{4}}{E I}, \quad K_{p}=\frac{k_{p} L^{2}}{E I}, \quad S=\frac{A L^{2}}{I}, \quad \tau=\frac{e_{0} a}{L}, \quad F=\frac{P L^{2}}{E I}
$$

Based on the introduced variables, the nondimensional form of the governing equations are reformulated as,

$$
\begin{gathered}
\left(S+\theta^{2}\right) \frac{\partial^{2} U_{0}}{\partial \beta^{2}}+(S \theta) \frac{\partial W_{0}}{\partial \beta}-\theta \frac{\partial^{3} W_{0}}{\partial \beta^{3}}=0 \\
(S \theta) \frac{\partial U_{0}}{\partial \beta}-\theta \frac{\partial^{3} U_{0}}{\partial \beta^{3}}+\left(S \theta^{2}+K_{w}\right) W_{0}+\left(S \alpha^{\prime} \Delta T-K_{p}-\tau^{2} K_{w}\right) \frac{\partial^{2} W_{0}}{\partial \beta^{2}}+ \\
+\left(1+\tau^{2} K_{p}-\tau^{2} S \alpha^{\prime} \Delta T\right) \frac{\partial^{4} W_{0}}{\partial \beta^{4}}-\tau^{2} F \frac{\partial^{4} W_{0}}{\partial \beta^{4}}+F \frac{\partial^{2} W_{0}}{\partial \beta^{2}}=0
\end{gathered}
$$

Employing the numerical method of differential quadrature and applying the proper boundary conditions, an eigenvalue problem is achieved. Solving this eigenvalue problem leads to obtaining the critical buckling loads.

\section{NUMERICAL SOLUTION}

Among the various numerical solutions could be used to solve the governing equations, differential quadrature method employed here. This method requires a low computational cost to solve initial and boundary value problems with a significant accuracy (Bellman and Casti, 1971; Bellman et al., 
1972; Shu and Richards, 1992; Jin et al., 2001). In this method, the problem domain is divided into $N$ points. The value of the $r^{\text {th }}$ order derivative of the function $f$ at each point of the domain is equal to a linear summation of the weighted function values at all points of the domain.

$$
f_{x}^{(r)}\left(x_{i}\right)=\sum_{j=1}^{N} B_{i j}^{(r)} f\left(x_{j}\right) \quad, \quad i=1,2, \cdots, N
$$

$B_{i j}^{(r)}$ are the $r^{\text {th }}$ order of the weighting coefficients. To calculate these coefficients, a set of test functions can be used. The weighting coefficients related to the first order derivative of the function $f$ can be calculated as follows (Bert and Malik, 1996),

$$
B_{i j}^{(1)}=\left\{\begin{array}{ll}
\frac{1}{L} \frac{M\left(x_{i}\right)}{\left(x_{i}-x_{j}\right) M\left(x_{j}\right)} & i \neq j \\
-\sum_{\substack{j=1 \\
j \neq i}}^{N} B_{i j}^{(1)} & i=j
\end{array} \quad M\left(x_{i}\right)=\prod_{\substack{j=1 \\
j \neq i}}^{N}\left(x_{i}-x_{j}\right)\right.
$$

The recursive relation to calculate the weighting coefficients of the higher order derivatives is given as,

$$
\left[B^{(m)}\right]=\left[B^{(m-1)}\right]\left[B^{(1)}\right] \quad, \quad m=2,3, \cdots, N
$$

Also, a set of cosine type (the Gauss-Lobatto-Chebyshev) grid distribution points is given by (Bert and Malik, 1996),

$$
\frac{x_{i}}{L}=\frac{1}{2}\left\{1-\cos \left[\frac{(i-1) \pi}{(N-1)}\right]\right\} \quad, \quad i=1,2, \cdots, N
$$

Herein, a new version of DQ method is employed which has been proposed by Karami and Malekzadeh (2002). Shortly, their methodology would overcome the difficulties encountered in boundary conditions implementations of fourth-order differential equations for the DQ analysis of beam structures. Using this method and satisfying the boundary conditions yield to find the critical buckling load as well as the critical buckling temperature. For the sake of brevity, more details of the DQ analysis are not presented here.

\section{NUMERICAL RESULTS}

In this section, numerical results are presented for the buckling behavior of CCNTs resting on an elastic foundation. The nondimensional critical buckling temperature and loads are determined for different values of the small scaling parameter. Three different boundary conditions including fully simply supported (S-S), simply-clamped supported (S-C) and fully clamped (C-C) are considered. 
In the first step, the accuracy and convergence study of the present method is demonstrated. Due to the lack of nonlocal solution for curved nanotubes, comparisons with solution of straight CNTs are carried out. Then, further analyses in terms of different parameters are presented. In all of the solutions, the Poisson's ratio and the thermal expansion coefficient are assumed to be $\nu=0.3$, and $\alpha_{t h}=1.1 \times 10^{-6}$, respectively. Also, the length to the thickness ratio of the nanotubes is assumed to be 10 with a nanotube length of $10 \mathrm{~nm}$, unless otherwise specified.

\subsection{Validation of the proposed approach}

The convergence behavior of the proposed method is demonstrated in Table 1. The nondimensional critical buckling loads of the straight nanotubes for three different values of the nonlocal parameters are presented. The validation is made for simply supported nanotubes. Reddy (2007) calculated the nondimensional critical buckling load based on the nonlocal Euler-Bernoulli beam theory. By comparing our results with the solution of Reddy (2007), it can be observed that with only seven nodes $(N)$, accurate results with a fast convergence behavior are achieved. This shows the efficiency of the DQ method in solving this kind of problems.

\begin{tabular}{ccccc}
\hline \hline$e_{0} a(\mathrm{~nm})$ & $N=6$ & $N=7$ & $N=8$ & Reddy (2007) \\
\hline 0 & 9.8673 & 9.8697 & 9.8696 & 9.8696 \\
1 & 8.9811 & 8.9831 & 8.9830 & 8.9830 \\
2 & 7.0749 & 7.0761 & 7.0761 & 7.0761 \\
\hline \hline
\end{tabular}

Table 1: The convergence of the nondimensional critical buckling loads of a straight CNT for different nonlocal parameters $\left(\Delta T=K_{w}=K_{p}=0\right)$.

Moreover, to further validate our approach, a comparison is made between the present results and those reported by Wang et al. (2010). Wang et al. (2010) analytically obtained the dimensionless critical buckling temperature of nanotubes based on the nonlocal Timoshenko beam theory. The dimensionless critical buckling temperature is given by,

$$
\Delta T^{N o n}=S \alpha_{t h} \Delta T
$$

In Table 2, the nondimensional critical buckling temperature of a simply supported straight CNT for three buckling mode numbers of 1, 5, and 10 are presented. The length to diameter (thickness) ratio is $L / d=100$ with the nanotube length of $100 \mathrm{~nm}$. Also, the nonlocal parameter is assumed to be $e_{0} a=1 \mathrm{~nm}$.

\begin{tabular}{ccc}
\hline \hline Mode No. & Present & Wang et al. (2010) \\
\hline 1 & 3.9478 & 3.9458 \\
5 & 98.69 & 97.43 \\
10 & 393.5 & 381.5 \\
\hline \hline
\end{tabular}

Table 2: The nondimensional critical buckling temperature of a straight CNT for three different buckling modes. 
It is seen that the results are very close to those obtained by Wang et al. (2010) in the case of modes 1 and 5, while the discrepancy for the higher mode of 10 is in an acceptable range of $3 \%$. This means that employing the nonlocal Euler-Bernoulli beam theory is quite reasonable for practical purposes.

Since no similar results have been found for the case of nonlocal buckling of curved carbon nanotubes, a comparison is carried out with the solution of Matsunaga (1996) presented for classical arch theory as shown in Table 3. The small scale parameter is set to be zero and the nondimensional critical buckling loads of CCNTs with different geometry parameters are determined. Again, the developed results are in good agreement with the existing numerical solution which verifies the reliability and validity of the present approach to predict the stability behavior of CCNTs.

\begin{tabular}{cccc}
\hline \hline$L / d$ & $L / R$ & Present & Matsunaga (1996) \\
\hline & 0.00 & 9.8696 & 9.8696 \\
2 & 0.10 & 9.8476 & 9.8568 \\
& 0.20 & 9.7816 & 9.8193 \\
& 0.30 & 9.6723 & 9.7581 \\
& 0.40 & 9.5205 & 9.6732 \\
\hline \multirow{2}{*}{5} & 0.00 & 9.8696 & 9.8696 \\
& 0.25 & 9.7430 & 9.8054 \\
& 0.50 & 9.3681 & 9.6179 \\
\hline \multirow{2}{*}{10} & 0.00 & 9.8696 & 9.6090 \\
& 0.25 & 9.7445 & 9.5478 \\
& 0.50 & 9.3740 & 9.3643 \\
& 0.00 & 9.8696 & 9.8035 \\
& 0.25 & 9.7449 & 9.7413 \\
\hline \hline
\end{tabular}

Table 3: The local nondimensional critical buckling loads of a simply supported CCNT $\left(e_{0} a=0\right)$.

At the next step, comparisons with the DQ Euler-Bernoulli beam solution of Murmu and Pradhan (2010) for the critical buckling loads of straight nanobeams are carried out. To clarify the comparison, a nondimensional parameter may be defined as

$$
F_{\text {scale }}=\frac{F_{\text {Nonlocal }}}{F_{\text {Local }}}
$$

where $F_{\text {Nonlocal }}$ and $F_{\text {Local }}$ represent the nondimensional critical buckling load for straight nanotubes using nonlocal and classic theories, respectively. In Figure 2, the variation of the dimensionless parameter of $F_{\text {Scale }}$ is depicted in terms of the nonlocal parameter, $e_{0} a$. As it can be observed, both results are matched quite well. 


\subsection{Effects of curvature and nonlocal parameter}

To consider the effects of curvature and the small scaling parameter on the stability of CCNTs, the dimensionless critical buckling temperature and loads for different angles of curvature and various nonlocal parameters are determined and listed in Tables 4 and 5, respectively. The results are shown for three different types of boundary conditions.

The trend of data in both of the Tables 4 and 5 are identical. The results indicate that with increasing the nonlocal parameter, the corresponding nondimensional critical buckling parameter decreases for all types of the boundary condition.

The reason is that the length of nanotube and consequently the flexibility of the nanotube are increased by increasing the angle of the curvature. However, the behavior is somehow different for fully clamped boundary condition. Moreover, it is worth noting that the stability of the curved nanotubes improves for end supports with a higher stiffness.

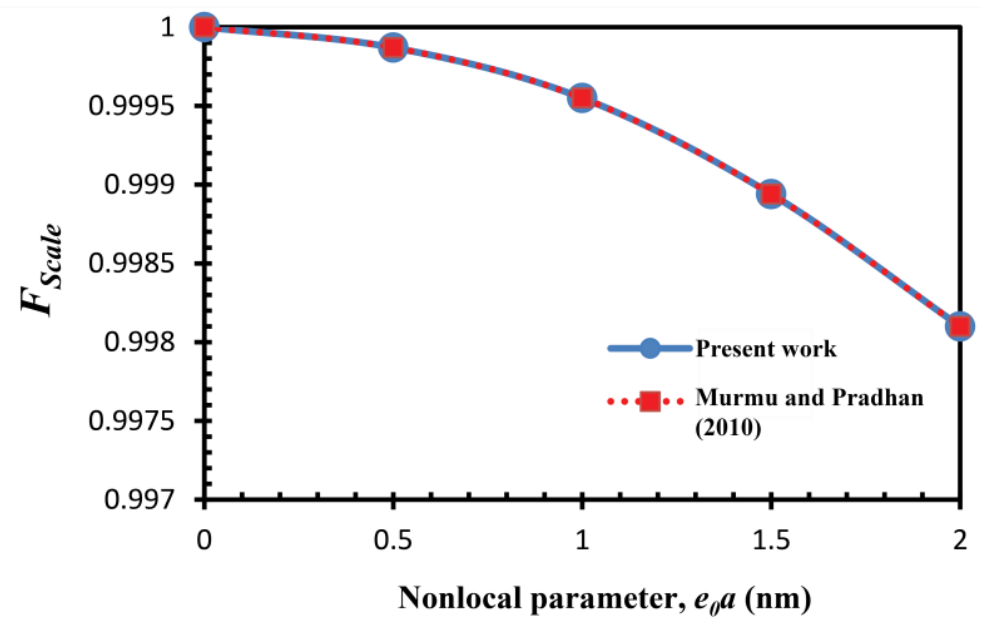

Figure 2: Variation in the ratio of the dimensionless nonlocal to the local buckling load vs. nonlocal parameter

$$
\left(L / d=100, L=100 \mathrm{~nm}, \theta=0, \Delta T=25, K_{w}=K_{p}=0\right) \text {. }
$$

\subsection{Influence of the elastic medium surrounding the CCNT}

To illustrate the effect of the elastic medium surrounding the CCNT, the nondimensional critical buckling temperature for various values of Winkler and Pasternak parameters are presented (Figures 3 and 4). The nondimensional critical buckling temperature as a function of Winkler elastic modulus for three different boundary conditions is demonstrated in Figure 3.

It can be observed that the nondimensional critical buckling temperature dependency on Winkler parameter decreases when the elastic foundation becomes stiffer. It is also seen that the influence of end-support stiffness on the nondimensional critical buckling temperature is higher than the Winkle parameter.

Figure 4 represents the dependency of the nondimensional critical buckling temperature on the Pasternak elastic modulus. As illustrated in the Figure, the dimensionless critical buckling temperature increases linearly by increasing the Pasternak elastic parameter for all types of boundary 


\begin{tabular}{|c|c|c|c|c|c|c|}
\hline \multirow[t]{2}{*}{$e_{0} a(\mathrm{~nm})$} & & \multicolumn{5}{|c|}{$\theta$} \\
\hline & & 0 & 0.5 & 1 & 1.5 & 2 \\
\hline & S-S & & & & & \\
\hline 0 & & 3.9478 & 3.7498 & 3.1864 & 2.3497 & 1.3928 \\
\hline 0.5 & & 3.8528 & 3.6595 & 3.1097 & 2.2931 & 1.3593 \\
\hline 1 & & 3.5932 & 3.4129 & 2.9001 & 2.1386 & 1.2677 \\
\hline 1.5 & & 3.2305 & 3.0684 & 2.6074 & 1.9227 & 1.1397 \\
\hline \multirow[t]{2}{*}{2} & & 2.8304 & 2.6884 & 2.2845 & 1.6846 & 0.9986 \\
\hline & $\mathrm{S}-\mathrm{C}$ & & & & & \\
\hline 0 & & 8.0763 & 7.8771 & 7.3047 & 6.4343 & 5.3898 \\
\hline 0.5 & & 7.6882 & 7.4986 & 6.9537 & 6.1251 & 5.1308 \\
\hline 1 & & 6.7196 & 6.5539 & 6.0776 & 5.3534 & 4.4842 \\
\hline 1.5 & & 5.5534 & 5.4165 & 5.0229 & 4.4243 & 3.7059 \\
\hline \multirow[t]{2}{*}{2} & & 4.4679 & 4.3577 & 4.0411 & 3.5595 & 2.9814 \\
\hline & C-C & & & & & \\
\hline 0 & & 15.7914 & 23.2170 & 31.4938 & 30.5041 & 29.1644 \\
\hline 0.5 & & 14.3728 & 21.0589 & 26.2032 & 25.3798 & 24.2651 \\
\hline 1 & & 11.3217 & 16.3740 & 17.4227 & 16.8752 & 16.1340 \\
\hline 1.5 & & 8.3629 & 11.3947 & 11.1792 & 10.8279 & 10.3524 \\
\hline 2 & & 6.1227 & 7.5879 & 7.4444 & 7.2105 & 6.8938 \\
\hline
\end{tabular}

Table 4: The dimensionless critical buckling temperature for different angles of curvature and nonlocal parameters $\left(k_{w}=k_{p}=0\right)$.

\begin{tabular}{|c|c|c|c|c|c|c|}
\hline \multirow[t]{2}{*}{$e_{0} a(\mathrm{~nm})$} & & \multicolumn{5}{|c|}{$\theta$} \\
\hline & & 0 & 0.5 & 1 & 1.5 & 2 \\
\hline & S-S & & & & & \\
\hline 0 & & 9.8696 & 9.3745 & 7.9659 & 5.8743 & 3.4820 \\
\hline 0.5 & & 9.6319 & 9.1487 & 7.7741 & 5.7328 & 3.3982 \\
\hline 1 & & 8.9830 & 8.5324 & 7.2504 & 5.3466 & 3.1692 \\
\hline 1.5 & & 8.0762 & 7.6710 & 6.5184 & 4.8068 & 2.8493 \\
\hline \multirow[t]{2}{*}{2} & & 7.0761 & 6.7211 & 5.7112 & 4.2116 & 2.4965 \\
\hline & S-C & & & & & \\
\hline 0 & & 20.1907 & 19.6928 & 18.2618 & 16.0857 & 13.4745 \\
\hline 0.5 & & 19.2205 & 18.7465 & 17.3843 & 15.3127 & 12.8270 \\
\hline 1 & & 16.7989 & 16.3846 & 15.1941 & 13.3834 & 11.2106 \\
\hline 1.5 & & 13.8836 & 13.5412 & 12.5572 & 11.0608 & 9.2648 \\
\hline \multirow[t]{2}{*}{2} & & 11.1697 & 10.8943 & 10.1026 & 8.8987 & 7.4535 \\
\hline & C-C & & & & & \\
\hline 0 & & 39.4784 & 58.0424 & 78.7344 & 76.2602 & 72.9109 \\
\hline 0.5 & & 35.9321 & 52.6472 & 65.5079 & 63.4493 & 60.6626 \\
\hline 1 & & 28.3043 & 40.9350 & 43.5567 & 42.1880 & 40.3350 \\
\hline 1.5 & & 20.9073 & 28.4867 & 27.9481 & 27.0698 & 25.8809 \\
\hline 2 & & 15.3068 & 18.9697 & 18.6111 & 18.0262 & 17.2345 \\
\hline
\end{tabular}

Table 5: The dimensionless critical buckling load for different angles of curvature and nonlocal parameters

$$
\left(\Delta T=K_{w}=K_{p}=0\right) \text {. }
$$


conditions studied here. Moreover, by comparing Figures 3 and 4, it can be concluded that the effect of the Pasternak elastic constant on the nondimensional critical buckling temperature is higher than the effect of the Winkler parameter.

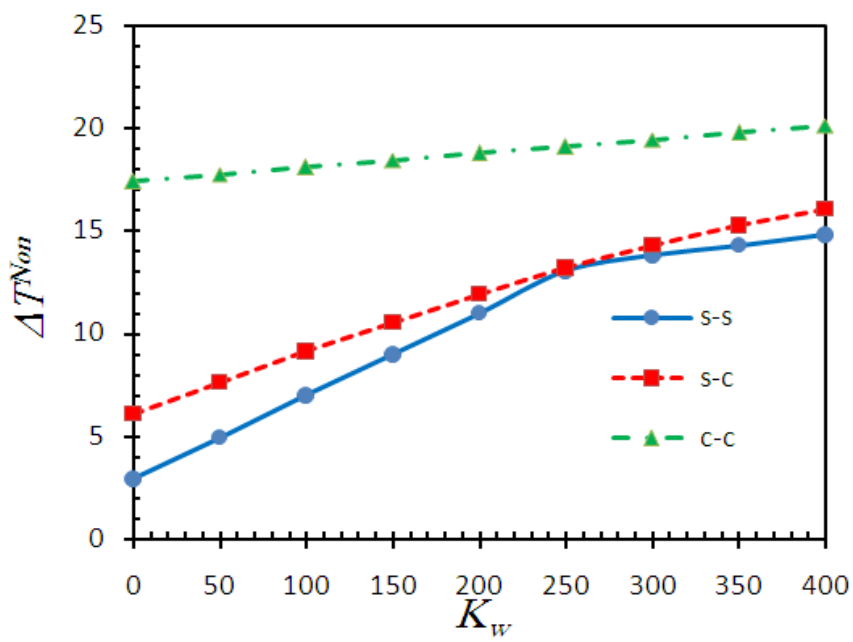

Figure 3: Dimensionless critical buckling temperature of the CCNT vs. Winkler parameter of the elastic medium

$$
\left(\theta=1, e_{0} a=1, K_{p}=0\right) .
$$

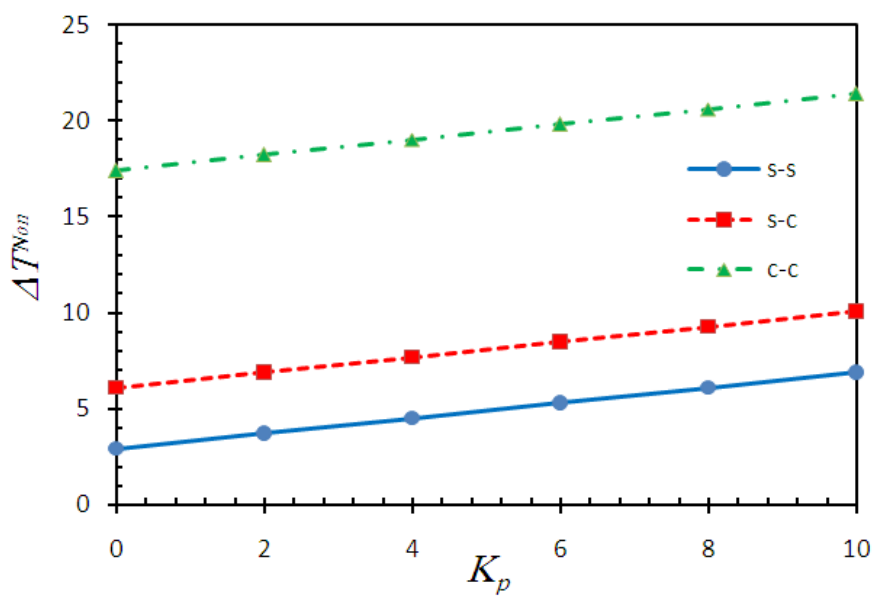

Figure 4: The nondimensional critical buckling temperature of the CCNT vs. Pasternak parameter of the elastic medium $\left(\theta=1, e_{0} a=1, K_{w}=0\right)$.

\subsection{The effect of temperature change}

In order to study the effects of temperature on the buckling of curved CNTs, a nondimensional thermal ratio parameter, $\lambda_{\text {thermal }}$ is defined as follows,

$$
\lambda_{\text {thermal }}=\frac{F_{\text {thermal }}}{F_{\text {Non-thermal }}}
$$


where $F_{\text {thermal }}$ and $F_{\text {Non-thermal }}$ represent the dimensionless critical buckling load when the influence of temperature change is considered or neglected, respectively. The variation of the nondimensional parameter $\lambda_{\text {thermal }}$ with respect to the temperature is presented in Figure 5 for different end supports.

As it is clear in Figure 5, for all types of boundary conditions considered, the thermal ratio $\lambda_{\text {thermal }}$ reduces with increasing the temperature change. In other words, with increasing the temperature, the nondimensional critical buckling load reduces. Furthermore, the slope of $\lambda_{\text {thermal }}$ versus temperature change increases as the end supports become stiffer.

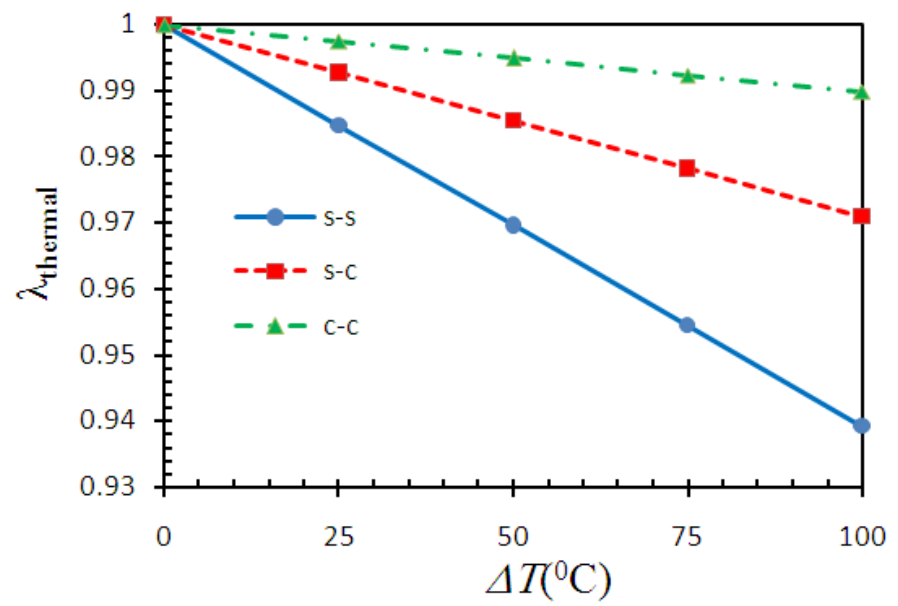

Figure 5: Thermal ratio parameter of CCNTs vs. the temperature change $\left(\theta=1, e_{0} a=1, K_{w}=K_{p}=0\right)$.

\section{CONCLUSIONS}

Thermal buckling analysis of CCNTs embedded in an elastic medium is investigated using the differential quadrature method. The governing equations are derived by combining thin curved beams and nonlocal elasticity theories. Employing the powerful numerical method of differential quadrature, the critical buckling temperature and loads are calculated for different types of boundary conditions. The elastic medium around the nanotube is modeled as the two-parameter Winkler-Pasternak foundation. It is found that the employed method demonstrate fast rate of convergence with very accurate results compared with the available solutions in literature which exhibits efficiency of the methodology. The effects of various parameters such as nonlocal parameter, angle of the nanotube curvature, Winkler and Pasternak moduli of the elastic medium and temperature change on the critical buckling load and temperature are successfully studied. The present results may be used as benchmark solutions for future researches.

The results reveal that the curvature of the nanotube has a considerable effect on the critical buckling load and temperature. Surprisingly, this effect is not identical for all sorts of boundary conditions. In the case of S-S and S-C supports, the dimensionless critical buckling load and temperature decrease as the angle of curvature increases. However, for fully clamped supports, the dimensionless critical buckling load and temperature increase up to a certain angle of curvature but then reduce due to further changes of the curvature angle. Moreover, it is shown that the stability of the curved nanotube is improved by increasing the Pasternak and Winkler elastic parameters. Also, 
the dimensionless critical buckling temperature reduces as the nonlocal parameter and the temperature increases.

\section{References}

Asemi, S.R., Farajpour, A., Borghei, M., Hassani, A.H., (2014). Thermal effects on the stability of circular graphene sheets via nonlocal continuum mechanics. Latin American Journal of Solids and Structures 11: 704-724.

Bellman, R., Casti, J., (1971). Differential quadrature and long-term integration. Journal of Mathematical Analysis and Applications 34: 235-238.

Bellman, R., Kashef, B., Casti, J., (1972). Differential quadrature: a technique for the rapid solution of nonlinear partial differential equations. Journal of Computational Physics 10: 40-52.

Bert, C.W., Malik, M., (1996). Differential quadrature method in computational mechanics: a review. Applied Mechanics Reviews 49: 1-28.

Cao, G., Chen, X., (2006). Buckling of single-walled carbon nanotubes upon bending: Molecular dynamics simulations and finite element method. Physical Review B 73: 155435.

Civalek, Ö., Demir, C., (2011). Buckling and bending analyses of cantilever carbon nanotubes using the euler-bernoulli beam theory based on non-local continuum model. Asian Journal of Civil Engineering (Building and Housing) 12: 651661.

Dai, H., Hafner, J.H., Rinzler, A.G., Colbert, D.T., Smalley, R.E., (1996). Nanotubes as nanoprobes in scanning probe microscopy. Nature 384: 147-150.

Eringen, A.C., (1983). On differential equations of nonlocal elasticity and solutions of screw dislocation and surface waves. Journal of Applied Physics 54: 4703-4710.

Falvo, M., Clary, G., Taylor, R., Chi, V., Brooks, F., Washburn, S., Superfine, R., (1997). Bending and buckling of carbon nanotubes under large strain. Nature 389: 582-584.

Friedman, R.S., McAlpine, M.C., Ricketts, D.S., Ham, D., Lieber, C.M., (2005). Nanotechnology: High-speed integrated nanowire circuits. Nature 434: 1085-1085.

Iijima, S., Brabec, C., Maiti, A., Bernholc, J., (1996). Structural flexibility of carbon nanotubes. The Journal of chemical physics 104: 2089-2092.

Jiang, H., Hwang, K., Liu, B., Huang, Y., (2004). Thermal expansion of single wall carbon nanotubes. Journal of engineering materials and technology 126: 265-270.

Jin, Z., Pramoda, K., Xu, G., Goh, S.H., (2001). Dynamic mechanical behavior of melt-processed multi-walled carbon nanotube/poly (methyl methacrylate) composites. Chemical Physics Letters 337: 43-47.

Karami, G., Malekzadeh, P., (2002). A new differential quadrature methodology for beam analysis and the associated differential quadrature element method. Computer Methods in Applied Mechanics and Engineering 191: 3509-3526.

Kulathunga, D., Ang, K., Reddy, J., (2010). Molecular dynamics analysis on buckling of defective carbon nanotubes. Journal of Physics: Condensed Matter 22: 345301.

Li, M., Kang, Z., Yang, P., Meng, X., Lu, Y., (2013). Molecular dynamics study on buckling of single-wall carbon nanotube-based intramolecular junctions and influence factors. Computational Materials Science 67: 390-396.

Liew, K., Wong, C., He, X., Tan, M., Meguid, S., (2004). Nanomechanics of single and multiwalled carbon nanotubes. Physical review B 69: 115429 .

Liu, L., Liu, F., Zhao, J., (2014). Curved carbon nanotubes: From unique geometries to novel properties and peculiar applications. Nano Research 7: 626-657.

Liu, P., Zhang, Y., Lu, C., Lam, K., (2004). Tensile and bending properties of double-walled carbon nanotubes. Journal of Physics D: Applied Physics 37: 2358. 
Malekzadeh, P., Mohebpour, S., Heydarpour, Y., (2012). Nonlocal effect on the free vibration of short nanotubes embedded in an elastic medium. Acta Mechanica 223: 1341-1350.

Matsunaga, H., (1996). In-plane vibration and stability of shallow circular arches subjected to axial forces. International Journal of Solids and Structures 33: 469-482.

Murmu, T., Adhikari, S., (2011). Axial instability of double-nanobeam-systems. Physics Letters A 375: $601-608$.

Murmu, T., Pradhan, S., (2010). Thermal effects on the stability of embedded carbon nanotubes. Computational Materials Science 47: 721-726.

Narendar, S., Gopalakrishnan, S., (2012). Nonlocal continuum mechanics formulation for axial, flexural, shear and contraction coupled wave propagation in single walled carbon nanotubes. Latin American Journal of Solids and Structures 9: 497-513.

Ozaki, T., Iwasa, Y., Mitani, T., (2000). Stiffness of single-walled carbon nanotubes under large strain. Physical review letters 84: 1712 .

Postma, H.W.C., Teepen, T., Yao, Z., Grifoni, M., Dekker, C., (2001). Carbon nanotube single-electron transistors at room temperature. Science 293: 76-79.

Qatu, M.S., (2004). Vibration of laminated shells and plates. Elsevier, Academic Press, (Oxford).

Rastgo, A., Shafie, H., Allahverdizadeh, A., (2005). Instability of curved beams made of functionally graded material under thermal loading. International Journal of Mechanics and Materials in Design 2: 117-128.

Reddy, J., (2007). Nonlocal theories for bending, buckling and vibration of beams. International Journal of Engineering Science 45: 288-307.

Ruoff, R.S., Lorents, D.C., (1995). Mechanical and thermal properties of carbon nanotubes. Carbon 33: $925-930$.

Sedighi, H.M., Daneshmand, F., Yaghootian, A., (2014). Application of iteration perturbation method in studying dynamic pull-in instability of micro-beams. Latin American Journal of Solids and Structures 11: 1078-1090.

Setoodeh, A., Khosrownejad, M., Malekzadeh, P., (2011). Exact nonlocal solution for postbuckling of single-walled carbon nanotubes. Physica E: Low-dimensional Systems and Nanostructures 43: 1730-1737.

Shu, C., Richards, B.E., (1992). Application of generalized differential quadrature to solve two-dimensional incompressible Navier-Stokes equations. International Journal for Numerical Methods in Fluids 15: 791-798.

Şimşek, M., Yurtcu, H., (2013). Analytical solutions for bending and buckling of functionally graded nanobeams based on the nonlocal Timoshenko beam theory. Composite Structures 97: 378-386.

Tans, S.J., Verschueren, A.R., Dekker, C., (1998). Room-temperature transistor based on a single carbon nanotube. Nature 393: 49-52.

Thai, H-T., (2012). A nonlocal beam theory for bending, buckling, and vibration of nanobeams. International Journal of Engineering Science 52: 56-64.

Thai, H-T., Vo, T.P., (2012). A nonlocal sinusoidal shear deformation beam theory with application to bending, buckling, and vibration of nanobeams. International Journal of Engineering Science 54: 58-66.

Tombler, T.W., Zhou, C., Alexseyev, L., Kong, J., Dai, H., Liu, L., Jayanthi, C., Tang, M., Wu, S-Y., (2000). Reversible electromechanical characteristics of carbon nanotubes under local-probe manipulation. Nature 405: 769-772.

Tounsi, A., Semmah, A., Bousahla, A.A., (2013). Thermal buckling behavior of nanobeams using an efficient higherorder nonlocal beam theory. Journal of Nanomechanics and Micromechanics 3: 37-42.

Wang, L., Ni, Q., Li, M., Qian, Q., (2008). The thermal effect on vibration and instability of carbon nanotubes conveying fluid. Physica E: Low-dimensional Systems and Nanostructures 40: 3179-3182.

Wang, Y-Z., Li, F-M., Kishimoto, K., (2010). Scale effects on thermal buckling properties of carbon nanotube. Physics Letters A 374: 4890-4893.

Wang, Y., Wang, X-x., Ni, X-g., Wu, H-a., (2005). Simulation of the elastic response and the buckling modes of singlewalled carbon nanotubes. Computational Materials Science 32: 141-146. 
Waters, J., Guduru, P., Jouzi, M., Xu, J., Hanlon, T., Suresh, S., (2005). Shell buckling of individual multiwalled carbon nanotubes using nanoindentation. Applied Physics Letters 87: 103109.

Yakobson, B.I., Brabec, C., Bernholc J., (1996). Nanomechanics of carbon tubes: instabilities beyond linear response. Physical review letters 76: 2511-2514. 